\title{
Identification of serum proteome signatures of locally advanced and metastatic gastric cancer: a pilot study
}

Agata Abramowicz' , Anna Wojakowska', Agnieszka Gdowicz-Klosok¹, Joanna Polanska², Pawel Rodziewicz³, Pawel Polanowski ${ }^{1}$, Agnieszka Namysl-Kaletka ${ }^{1}$, Monika Pietrowska' ${ }^{1}$, Jerzy Wydmanski ${ }^{1}$ and Piotr Widlak ${ }^{{ }^{*}}$

\begin{abstract}
Background: The gastric cancer is one of the most common and mortal cancer worldwide. The initial asymptomatic development and further nonspecific symptoms result in diagnosis at the advanced stage with poor prognosis. Yet, no clinically useful biomarkers are available for this malignancy, and invasive gastrointestinal endoscopy remains the only reliable option at the moment. Hence, there is a need for discovery of clinically useful noninvasive diagnostic and/or prognostic tool as an alternative (or complement) for current diagnostic tools. Here we aimed to search for serum proteins characteristic for local and invasive gastric cancer.
\end{abstract}

Methods: Pre-treatment blood samples were collected from patients with diagnosed gastric adenocarcinoma at the different stage of disease: 35 patients with locally advanced cancer and 18 patients with metastatic cancer; 50 healthy donors were also included as a control group. The low-molecular-weight fraction of serum proteome (i.e., endogenous peptidome) was profiled by the MALDI-ToF mass spectrometry, and the whole proteome components were identified and quantified by the LC-MS/MS shotgun approach.

Results: Multicomponent peptidome signatures were revealed that allowed good discrimination between healthy controls and cancer patients, as well as between patients with locally advanced and metastatic cancer. Moreover, a LC-MS/MS approach revealed 49 serum proteins with different abundances between healthy donors and cancer patients (predominantly proteins associated with inflammation and acute phase response). Furthermore, 19 serum proteins with different abundances between patients with locally advanced and metastatic cancer were identified (including proteins associated with cytokine/chemokine response and metabolism of nucleic acids). However, neither peptidome profiling nor shotgun proteomics approach allowed detecting serum components discriminating between two subgroups of patients with local disease who either developed or did not develop metastases during follow-up.

Conclusions: The molecular differences between locally advanced and metastatic gastric cancer, as well as more obvious differences between healthy individuals and cancer patients, have marked reflection at the level of serum proteome. However, we have no evidence that features of pre-treatment serum proteome could predict a risk of cancer dissemination in patients treated due to local disease. Nevertheless, presented data confirmed potential applicability of a serum proteome signature-based biomarker in diagnostics of gastric cancer.

Keywords: Stomach cancer, Early diagnosis, Neoplasms, Proteomics, Biological markers

\footnotetext{
*Correspondence: piotr.widlak@io.gliwice.pl

${ }^{1}$ Maria Sklodowska-Curie Memorial Cancer Center and Institute

of Oncology, Gliwice, Poland

Full list of author information is available at the end of the article
} 


\section{Background}

Gastric cancer is the fourth most common cancer and the second leading cause of cancer-related death worldwide. This cancer especially afflicts populations of East Asia, Eastern Europe, and parts of Central and South America, and the morbidity rate is twice higher for men than for women [1]. The malignancy is associated with nonspecific symptoms or even asymptomatic development in its early stages, which often results in diagnosis at advanced stages. Stage of gastric cancer strongly correlates with poor prognosis. According to the National Cancer Data Base report, 5-year survival rate for stage IA was $78 \%$ and it dropped substantially at each stage to about $7 \%$ for patients diagnosed with stage IIIB or stage IV disease [2]. Thus, early diagnosis of gastric cancer might radically increase efficacy of treatment and improve prognosis for this fatal illness. At present the most efficient diagnostic tool for detection of gastric cancer remains a gastrointestinal endoscopy, yet this invasive technique is not suitable for large-scale screening. Unfortunately, there is no alternative non-invasive biomarkers available, because the commonly used gastrointestinal tumor markers like CEA, CA 19-9 or CA 72-4 are insufficient for early diagnosis of this cancer due to their low sensitivity and specificity (20-30\%) [3-5]. Majority of gastric cancer cases (above $90 \%$ ) are classified as adenocarcinomas. More recently four molecular subtypes of gastric adenocarcinoma were distinguished based on genomic profiling delivered thanks to the Cancer Genome Atlas project [6]. However, the knowledge on molecular heterogeneity and biology of this cancer, including its development and mechanisms of progression, remains rather limited yet. Hence, an urgent need for identification of clinically relevant biomarkers relates not only the early diagnosis but also prognosis and prediction of treatment outcome.

Clinical proteomics is an important approach to discovery of biomarkers of gastric cancer [7]. It is generally accepted that blood proteome is a promising source of novel biomarkers of this cancer, including particularly valuable markers for early detection of the disease and monitoring of response to the treatment [8]. Mass spectrometry-based profiling of the low-molecular-weight fraction of serum proteome, so called endogenous peptidome, revealed multi-peptide signatures with potential applicability in classification and diagnosis of different cancer types [9-13]. A few works have been published that explored MALDI/SELDI-based profiling of serum/ plasma petidome for diagnosis of gastric cancer, which proposed peptide signatures that allowed discriminating healthy donors and patients with gastric cancer, or signatures associated with a course of a disease [14-22]. Several components of such signatures were further identified as fragments of KNG1 [18], APOC1 and APOA2 [19], SAA
[20], TBB5 and TYB4 [22] or FIBA [23, 24]. More recently, a panel of biomarkers composed of serum proteins preselected based on preclinical mouse model (afamin, clusterin, VDBP and haptoglobin) has been validated to discriminate between gastric cancer patients and patient with benign gastric diseases [25]. Nevertheless, none of proposed serum proteome signatures of gastric cancer has been widely accepted and applied in clinical practice yet.

Here we aimed to characterize proteome features of pre-treatment serum associated with risk of metastasis of gastric cancer. Two types of proteomic analyses were performed: (1) the low-molecular-weight fraction of serum proteome was profiled by the MALDI-ToF mass spectrometry, (2) the whole proteome components were identified and quantified by LC-MS/MS after digestion with trypsin (a "shotgun proteomics" approach). Groups of previously untreated patients with locally advanced gastric cancer and metastatic disease were enrolled to this study (a matched group of healthy individuals was analyzed as a reference); such comprehensive proteomic analysis was performed in a group of Caucasians patients with gastric cancer for the first time. Serum proteome signature that differentiated between patients with locally advanced cancer and metastatic cancer was detected in this pilot study, yet features specific for patients with locally advanced disease at time of diagnosis who eventually developed metastases were not observed at this level.

\section{Methods}

\section{Characteristics of patient groups}

Fifty-three patients with previously untreated biopsyproven gastric adenocarcinoma were qualified into this study: 35 patients with locally advanced cancer, including 16 patients with metastasis developed during therapy or follow-up and 19 patients with no detected metastasis during follow-up, and 18 patients with metastatic cancer. The latter group consisted of four patients with distant spread to single organ and 14 patients with spread to multiple organs; involved organs included peritoneum (13 cases), liver (eight cases), lung (three cases), other locations (eight cases). In general, inclusion criteria involved: Eastern Cooperative Oncology Group (ECOG) performance status of $0-2$, age 20-85 years, serum creatinine level $<1.5 \mathrm{mg} / \mathrm{dl}$, serum bilirubin level $<2.0 \mathrm{mg} / \mathrm{dl}$, a granulocyte count $>1500$ cells $/ \mu \mathrm{l}$ and a platelet count $>100,000$ cells/ $\mu$ l, while exclusion criteria involved: previous malignancy, previous surgery, radiotherapy or chemotherapy. The pretreatment staging based on physical examination, esophagogastroscopy with biopsies, CT of the abdomen and chest examination by X-ray or CT. Fifty sex- and age-matched disease-free donors were included as a control group. All study participants were Caucasians ( $65 \%$ men) with the age at the range $34-74$ years. 
Table 1 shows more detailed information about analyzed groups. The study was approved by the appropriate Ethics Committee, and all persons who were taking part in this study provided informed consent indicating their conscious and voluntary participation.

\section{Preparation of serum samples}

Pre-treatment blood was collected into a $5 \mathrm{ml}$ Vacutainer Tube (Becton-Dickinson), incubated for $30 \mathrm{~min}$ at room temperature to allow clotting and then centrifuged at $1000 \mathrm{~g}$ for $10 \mathrm{~min}$ to remove the clot. The serum was aliquoted and stored at $-70{ }^{\circ} \mathrm{C}$ until use.

\section{Profiling of the low-molecular-weight fraction of serum proteome}

Before analysis samples were diluted 1:5 with buffer containing $20 \%$ acetonitrile (ACN) and $25 \mathrm{mM}$ ammonium bicarbonate, and then filtered by centrifugation through Amicon Ultra units (50 kDa cut-off) for removing the abundant high-molecular weight proteins, particularly albumin. Immediately before analysis samples were desalted and concentrated by loading onto ZipTip C18 microcolumns (EMD Millipore), and then eluted with $1 \mu \mathrm{l}$ of matrix solution (saturated solution of alphacyano-4-hydroxy-cinnamic acid in $30 \% \mathrm{ACN} / \mathrm{H}_{2} \mathrm{O}$ and
$0.1 \%$ TFA) directly onto the $800 \mu \mathrm{m}$ AnchorChip $^{\text {TM }}$ (Bruker Daltonics) plate. The analysis was done by using an UltrafleXtreme MALDI-ToF mass spectrometer (Bruker Daltonics); the analyzer worked in the linear mode, and positive ions were recorded in the mass range between 1000 and 12,000 Da. The samples were spotted in duplicate and for each spot two spectra were acquired. Mass calibration was performed after every four samples using Protein Calibration Standard I (Bruker Daltonics). Randomization in blocks was used in spectra registration to avoid a possible batch effect. Afterwards the raw data was exported to TXT files and the spectral components were preprocessed using bioinformatics algorithms created in our group, which included alignment, detection and removal of outlier profiles by Dixon's Q test (single spectra were removed from about $5 \%$ of samples), averaging of technical repeats, baseline removal and normalization of the total ion current. The spectra smoothing, peak picking, binning and statistical analysis were performed using Spectrolyzer software (version 1.0.21.3590, MedicWave).

\section{LC-MS/MS analysis of serum proteome components}

Serum samples were reduced with $5 \mathrm{mM}$ dithiothreitol for $5 \mathrm{~min}$ at $95{ }^{\circ} \mathrm{C}$, then alkylated with $10 \mathrm{mM}$

Table 1 Characteristics of donor groups enrolled into the study

\begin{tabular}{|c|c|c|c|c|c|}
\hline \multirow[t]{2}{*}{ Group/parameter } & \multirow[t]{2}{*}{ Healthy control } & \multirow{2}{*}{$\begin{array}{l}\text { Cancer patients } \\
\text { (all cases) }\end{array}$} & \multicolumn{2}{|c|}{ Patients with locally advanced cancer } & \multirow{2}{*}{$\begin{array}{l}\text { Patients } \\
\text { with metastatic } \\
\text { cancer }\end{array}$} \\
\hline & & & No spread & Spread & \\
\hline Number (n) & 50 & 53 & 19 & 16 & 18 \\
\hline $\operatorname{Sex}(M / F)$ & $30 / 20$ & $37 / 16$ & $12 / 7$ & $13 / 3$ & $12 / 6$ \\
\hline Age (years) & 28-60 (median 50) & 34-74 (median 59) & 36-70 (median 59) & 34-73 (median 58) & 35-74 (median 60) \\
\hline \multicolumn{6}{|l|}{ Tumor location } \\
\hline Upper third & - & $15(28 \%)$ & $3(16 \%)$ & $6(37 \%)$ & $6(33 \%)$ \\
\hline Middle third & - & $31(59 \%)$ & $13(68 \%)$ & $9(57 \%)$ & $9(50 \%)$ \\
\hline Lower third & - & $7(13 \%)$ & $3(16 \%)$ & $1(6 \%)$ & $3(17 \%)$ \\
\hline \multicolumn{6}{|l|}{ Histological grade } \\
\hline $\mathrm{G} 1-\mathrm{G} 2$ & - & $16(30 \%)$ & $10(53 \%)$ & $3(19 \%)$ & $3(17 \%)$ \\
\hline G3 & - & $29(55 \%)$ & $8(42 \%)$ & 11 (69 \%) & $10(56 \%)$ \\
\hline Not specified & - & $8(15 \%)$ & $1(5 \%)$ & $2(12 \%)$ & $5(27 \%)$ \\
\hline \multicolumn{6}{|l|}{ Primary tumor } \\
\hline сT1-T3 & - & $50(94 \%)$ & $19(100 \%)$ & $16(100 \%)$ & $15(83 \%)$ \\
\hline cT4 & - & $3(6 \%)$ & $0(0 \%)$ & $0(0 \%)$ & $3(17 \%)$ \\
\hline \multicolumn{6}{|l|}{ Lymph node } \\
\hline $\mathrm{CNO}$ & - & 20 (38 \%) & $13(68 \%)$ & $5(31 \%)$ & $2(11 \%)$ \\
\hline $\mathrm{CN} 1-\mathrm{N} 3$ & - & $33(62 \%)$ & $6(32 \%)$ & $11(69 \%)$ & $16(89 \%)$ \\
\hline \multicolumn{6}{|l|}{ Metastasis (initial) } \\
\hline CMO & - & $35(66 \%)$ & 19 (100 \%) & 16 (100\%) & $0(0 \%)$ \\
\hline cM1 & - & $18(34 \%)$ & $0(0 \%)$ & $0(0 \%)$ & 18 (100\%) \\
\hline
\end{tabular}

Group of patients with locally advanced cancer at time of diagnosis were further split into subgroup where either no spread (control) or consecutive cancer dissemination/spread (distant metastasis) was detected 
iodoacetamide for $20 \mathrm{~min}$ in darkness at room temperature, and afterwards digested overnight at $37{ }^{\circ} \mathrm{C}$ with trypsin (Promega). The analysis was performed on Dionex UltiMate 3000 RSLC nanoLC System connected to Q Exactive Orbitrap mass spectrometer (Thermo Fisher Scientific); each sample was analyzed separately. Tryptic peptides ( $2.5 \mu \mathrm{g}$ of peptides) were separated on reverse phase Acclaim PepMap RSLC nanoViper C18 column $(75 \mu \mathrm{m} \times 25 \mathrm{~cm}, 2 \mu \mathrm{m}$ granulation) using the acetonitrile gradient (from 4 to $60 \%$, in $0.1 \%$ formic acid) at $30{ }^{\circ} \mathrm{C}$ and a flow rate of $250 \mathrm{~nL} / \mathrm{min}$ (for $230 \mathrm{~min}$ ). The spectrometer was operating in the data-dependent MS/MS mode with survey scans acquired at a resolution of 70,000 at m/z $200 \mathrm{Da}$ in MS mode and 17,500 at $\mathrm{m} / \mathrm{z} 200 \mathrm{Da}$ in MS2 mode, respectively. The spectra were recorded in the scan $\mathrm{m} / \mathrm{z}$ range $300-2000$ in the positive ion mode. Higher energy collisional dissociation (HCD) ion fragmentation was performed with normalized collision energies set to 25. Protein identification was performed using Swiss-Prot human database with a precision tolerance $10 \mathrm{ppm}$ for peptide masses and $0.05 \mathrm{Da}$ for fragment ion masses. The abundances of identified proteins were estimated using MaxQuant 1.4.1.1 software.

\section{Statistical and bioinformatics analyses}

For each component of MALDI mass profiles the comparison between groups of donors was performed using the Student's t test after logarithmic transformation of data. Multi-component classifiers were built and tested with the SVM-based approach using Spectrolyzer software (version 1.0.21.3590, MedicWave). Significance of differences in abundances of proteins quantified by LC-MS/MS were assessed using the $t$ test or the MannWhitney test depending on normality of data (type of distribution was estimated using the Shapiro-Wilk test, the Lilliefors test and the F test for homogeneity of variances), and the Nemenyi test for pairwise comparisons. In general, $p=0.05$ was selected as a statistical significance threshold except for MALDI profiling where the Bonferroni correction for multiple testing was applied. The Empirical Proteomic Ontology Knowledge Base (EPO-KB), which annotates registered $\mathrm{m} / \mathrm{z}$ values to known peptide/proteins [26], was employed to assign hypothetical identification of the spectra components ( $0.5 \%$ mass accuracy limit was allowed). List of genes corresponding to identified proteins was annotated at GO terms using gProfiler (http://biit.cs.ut.ee/gprofiler/); the significance of the term over-representation was assessed using the hypergeometric distribution test. In order to visualize functional relationships between identified proteins corresponding genes were annotated at the GeneMANIA Cytoscape plugin for pathway interaction networks (http://pages.genemania.org/plugin/).

\section{Results}

Mass profiles of the serum endogenous peptidome (the low-molecular-weight fraction of serum proteome) were characterized by MALDI-ToF spectrometry in the whole group of 53 patients with gastric cancer and 50 matched healthy donors. This analysis allowed us to asses overall degree of differences and similarities between subgroups of analyzed individuals. In general, 255 spectral components (peptide ions) were distinguished in the analyzed mass range (Fig. 1a), and abundances of 101 components revealed statistically significant variation among compared groups (after the Bonferroni correction against multiple testing). Table 2 presents numbers of serum peptidome components that differentiated particular groups of donors. The major differences in abundances of specific serum components were observed between cancer patients and healthy donors (about $39 \%$ of registered components reveled statistically significant differences). Large differences were also observed between patients with locally advanced cancer (all cases) and patients with metastatic cancer (about $9 \%$ of registered components reveled statistically significant differences); this is noteworthy that similar numbers of differentiating components were observed when patients with metastatic cancer were compared with both subgroups with local disease separately (i.e., group where distant spread of cancer was detected during follow-up and group without evidence of disease). Coherently, well performing classifiers could be built based on features of serum peptidome that separated compared groups of healthy donors and patients with locally advanced or metastatic cancer (the AUC measure for SVM-based classifier was above $90 \%$ in each case). In marked contrast, no statistically significant difference was detected when two subgroups of patients with locally advanced cancer (who either developed or not developed metastasis during follow-up) were compared (the AUC of SVM-based classifier was below $50 \%$ ). Figure $1 \mathrm{~b}$ presents examples of serum peptidome components with significantly different abundances among compared groups. This is noteworthy that among petidome components that differentiated both healthy controls from cancer patients and patients with locally advanced cancer from patients with metastatic disease there were several components that putatively corresponded to fragments of fibrinopeptide A (FIBA). These included components with registered $\mathrm{m} / \mathrm{z}$ value 1088.7, 5903.5 and 5916.6 Da significantly downregulated in serum of cancer patients, and components 1469.9 and 1626.0 Da with markedly higher abundances 


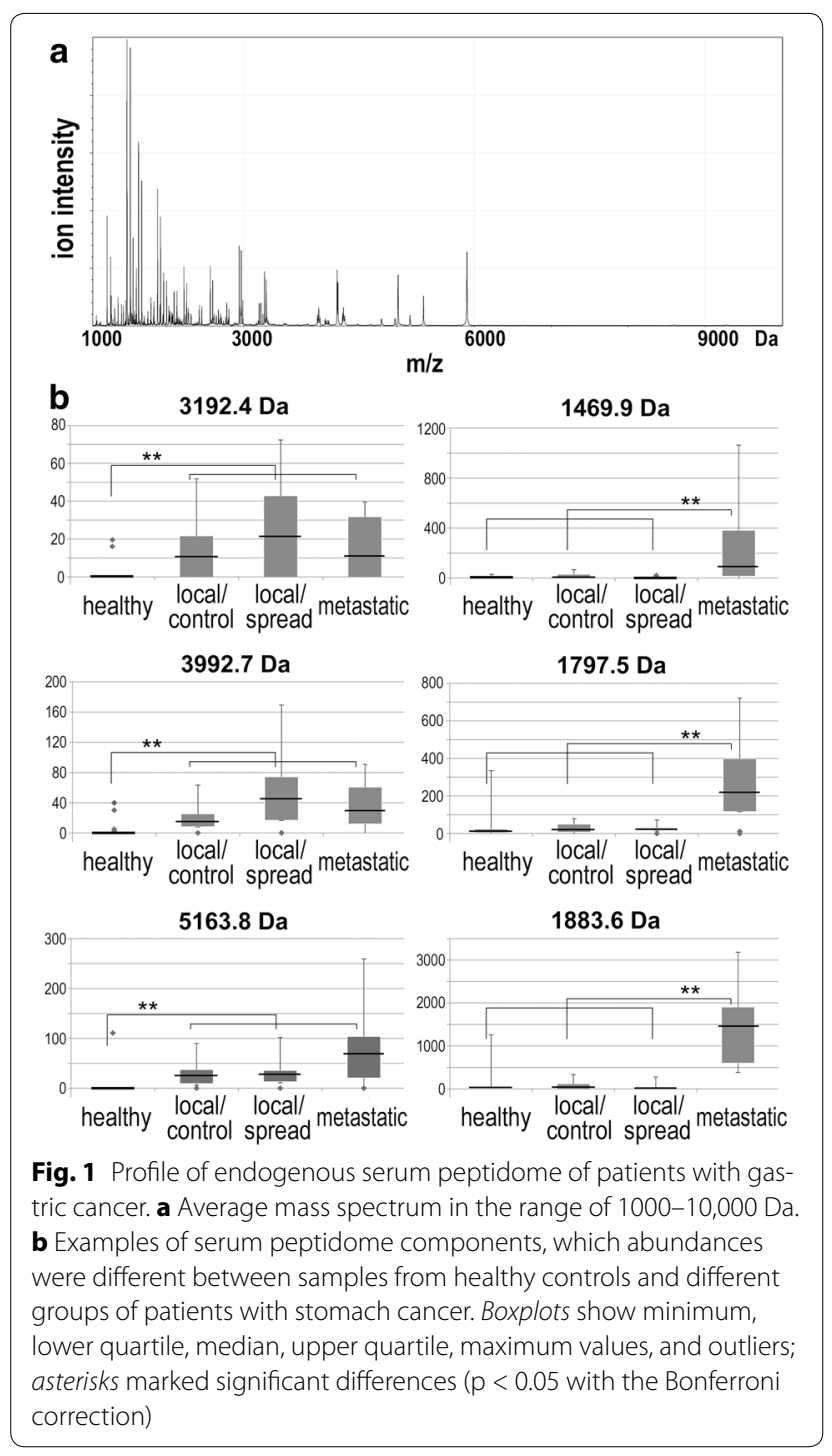

in serum of patients with metastatic disease than in patients with locally advanced cancer (see Fig. 1b; Additional file 1: Table S1). We concluded that molecular differences between locally advanced and metastatic gastric cancer (as well as differences between healthy individuals and patients with gastric cancer in general) have reflection at the level of serum peptidome. However, features of peptidome of pre-treatment serum could be unlikely applied for prognosis of the disease spread during/after the treatment.

In the second step we selected samples of 12 healthy donors and ten patients from each cancer subgroup to secure similar age (medians about 56 years) and proportion of sexes (ca. $80 \%$ of males) in compared subgroups. These samples were used for further analyses based on the shotgun LC-MS/MS approach. In general, about 450 proteins were identified in analyzed serum samples. Levels of 234 serum proteins were quantified in samples collected from each of 42 individuals, including 129 unique serum proteins not related to immunoglobulins (105 immunoglobulins and Ig-related proteins, as well as putative uncharacterized proteins, were excluded from further analyses); complete data are presented in the Additional file 1: Table S2. There were 49 serum proteins with abundances different between healthy donors and patients with gastric cancer ( $\mathrm{p}$ value $<0.05$; estimated FDR value $=13 \%$ ): 41 proteins were upregulated while eight proteins were downregulated in blood of cancer patients (proteins listed in Table 3; examples in Fig. 2). This is noteworthy that similar patterns of cancer-related upregulation or downregulation of serum proteins was observed in all there subgroups of cancer patients (even though smaller size of analyzed groups might reduce statistical significance of differences; see Additional file 1: Table S3). Moreover, there were 19 serum proteins with abundances different between patients with locally advanced and metastatic cancer ( $\mathrm{p}$ value $<0.05$; estimated FDR value $=34 \%$ ): three proteins were upregulated while 16 proteins were downregulated in blood of patients with metastatic disease. This is noteworthy that abundances of C-reactive protein and angiogenin generally upregulated in cancers samples further increased in metastatic samples, while abundances of carbonic anhydrase 1 generally downregulated in cancer samples further decreased

Table 2 Numbers of serum peptidome components with abundances different between compared groups of individuals

\begin{tabular}{llllll}
\hline Groups/differences & $\begin{array}{l}\text { Control vs. } \\
\text { cancer (all cases) }\end{array}$ & $\begin{array}{l}\text { Locally advanced vs. } \\
\text { metastatic cancer }\end{array}$ & $\begin{array}{l}\text { Local/no spread vs. } \\
\text { metastatic cancer }\end{array}$ & $\begin{array}{l}\text { Local/spread vs. } \\
\text { metastatic cancer }\end{array}$ & $\begin{array}{l}\text { Local/no spread vs. } \\
\text { local/spread cancer }\end{array}$ \\
\hline $\mathrm{n}$ & 50 vs. 53 & 35 vs. 18 & 19 vs. 18 & 16 vs. 18 & 19 vs. 16 \\
$p<0.05$ & 182 & 88 & 74 & 75 & 11 \\
FDR & $7 \%$ & $14 \%$ & $17 \%$ & $17 \%$ & $100 \%$ \\
$p<0.05 /$ Bonferroni & 101 & 23 & 11 & 16 & 0 \\
AUC (SVM) & 0.94 & 0.91 & 0.92 & 0.97 & 0.48 \\
\hline
\end{tabular}

Shown are numbers of differentiating components that reached threshold of statistical significance $\mathrm{p}=0.05$ (with corresponding FDR estimation) or threshold strengthened with the Bonferroni correction, and power of SVM classifier built of peptidome components (characterized by the AUC value) 
Table 3 Differentiating serum proteins

\begin{tabular}{|c|c|c|c|c|c|c|}
\hline \multirow[t]{2}{*}{ Protein name } & \multirow[t]{2}{*}{ Protein full name } & \multirow[t]{2}{*}{ Gene name } & \multicolumn{2}{|c|}{ Control/cancer } & \multicolumn{2}{|c|}{ Local/metastatic } \\
\hline & & & Ratio & p value & Ratio & $\mathrm{p}$ value \\
\hline $\mathrm{A} 1 \mathrm{AG} 1$ & Alpha-1-acid glycoprotein 1 & ORM1 & 0.67 & 0.0008 & 1.00 & 0.5824 \\
\hline A1AG2 & Alpha-1-acid glycoprotein 2 & ORM2 & 0.68 & 0.0006 & 1.00 & 0.7414 \\
\hline A1AT & Alpha-1-antitrypsin & SERPINA1 & 0.50 & $<0.0001$ & 1.03 & 0.6441 \\
\hline A1BG & Alpha-1B-glycoprotein & A1BG & 0.76 & 0.0072 & 1.04 & 0.5824 \\
\hline $\mathrm{A} 2 \mathrm{GL}$ & Leucine-rich alpha-2-glycoprotein & LRG1 & 0.46 & 0.0016 & 0.80 & 0.4414 \\
\hline AACT & Alpha-1-antichymotrypsin & SERPINA3 & 0.54 & 0.0007 & 0.97 & 0.9124 \\
\hline ADIPO & Adiponectin & ADIPOQ & 0.41 & 0.0092 & 1.60 & 0.3442 \\
\hline AFAM & Afamin & AFM & 1.30 & 0.0355 & 1.06 & 0.5824 \\
\hline ANGI & Angiogenin & ANG & 0.30 & 0.0465 & 0.24 & 0.0294 \\
\hline APOA1 & Apolipoprotein A-I & APOA1 & 0.47 & $<0.0001$ & 1.31 & 0.0235 \\
\hline APOC1 & Apolipoprotein C-I & APOC1 & 0.25 & $<0.0001$ & 1.52 & 0.1183 \\
\hline APOC3 & Apolipoprotein C-III & APOC3 & 1.82 & 0.0108 & 1.20 & 0.5824 \\
\hline APOE & Apolipoprotein E & APOE & 0.62 & 0.0040 & 1.01 & 0.6441 \\
\hline APOF & Apolipoprotein F & APOF & 0.64 & 0.0085 & 0.80 & 0.5235 \\
\hline APOM & Apolipoprotein M & APOM & 0.92 & 0.4275 & 1.51 & 0.0263 \\
\hline C1S & Complement $\mathrm{C}$ 1s subcomponent & C1S & 0.70 & 0.0016 & 1.22 & 0.0748 \\
\hline $\mathrm{CAH1}$ & Carbonic anhydrase 1 & CA1 & 2.96 & 0.0173 & 2.41 & 0.0143 \\
\hline CBG & Corticosteroid-binding globulin & SERPINA6 & 0.53 & 0.0117 & 1.08 & 0.7749 \\
\hline CD14 & Monocyte antigen CD14 & CD14 & 0.56 & 0.0033 & 1.21 & 0.1658 \\
\hline CERU & Ceruloplasmin & $C P$ & 0.67 & 0.0013 & 1.05 & 0.6129 \\
\hline CFAB & Complement factor B & CFB & 0.61 & 0.0006 & 1.11 & 0.6441 \\
\hline $\mathrm{CO} 2$ & Complement C2 & C2 & 0.64 & 0.0475 & 1.57 & 0.0679 \\
\hline CO4A & Complement C4-A & C4A & 0.87 & 0.0435 & 0.94 & 0.7749 \\
\hline $\mathrm{CO} 4 \mathrm{~B}$ & Complement C4-B & $\mathrm{C} 4 \mathrm{~B}$ & 0.73 & 0.0127 & 0.99 & 0.5526 \\
\hline CO5 & Complement C5 & C5 & 0.77 & 0.0085 & 0.91 & 0.8776 \\
\hline CO6 & Complement component C6 & $\mathrm{C} 6$ & 0.64 & 0.0013 & 0.97 & 0.8431 \\
\hline CO8G & Complement component C8 gamma & C8G & 0.66 & 0.0137 & 1.12 & 0.4679 \\
\hline $\operatorname{cog}$ & Complement component C9 & C9 & 0.42 & $<0.0001$ & 0.93 & 0.9124 \\
\hline CRP & C-reactive protein & CRP & $<0.01$ & 0.0389 & 0.15 & 0.0414 \\
\hline CXCL7 & Platelet basic protein & PPBP & 0.83 & 0.1516 & 1.53 & 0.0030 \\
\hline FETUA & Alpha-2-HS-glycoprotein & AHSG & 0.90 & 0.3095 & 1.34 & 0.0068 \\
\hline FHR1 & Complement factor H-related prot. 1 & CFHR1 & 0.66 & 0.0725 & 1.30 & 0.0366 \\
\hline GPX3 & Glutathione peroxidase 3 & GPX3 & 0.92 & 0.6065 & 1.47 & 0.0453 \\
\hline HBA & Hemoglobin subunit alpha & HBA1 & 2.02 & 0.0016 & 1.18 & 0.5824 \\
\hline HBB & Hemoglobin subunit beta & $\mathrm{HBB}$ & 2.26 & 0.0005 & 1.11 & 0.6441 \\
\hline $\mathrm{HBD}$ & Hemoglobin subunit delta & $\mathrm{HBD}$ & 4.59 & 0.0030 & 1.02 & 0.9608 \\
\hline HEMO & Hemopexin & HPX & 0.77 & 0.0061 & 1.00 & 0.9124 \\
\hline HGFA & Hepatocyte growth factor activator & HGFAC & 1.57 & 0.0868 & 1.74 & 0.0366 \\
\hline HGFL & Hepatocyte growth factor-like prot. & MST1 & 1.40 & 0.4048 & 3.28 & 0.0156 \\
\hline HPT & Haptoglobin & $\mathrm{HP}$ & 0.68 & 0.0014 & 1.02 & 0.7749 \\
\hline IC1 & Plasma protease $\mathrm{C} 1$ inhibitor & SERPING1 & 3.36 & 0.0001 & 0.21 & 0.0030 \\
\hline ITIH1 & Inter-alpha-trypsin inhib. heavy ch. 1 & $\mathrm{ITIH} 1$ & 0.77 & 0.0465 & 1.06 & 0.2099 \\
\hline ITIH3 & Inter-alpha-trypsin inhib. heavy ch. 3 & $\mathrm{ITIH} 3$ & 0.45 & 0.0001 & 1.05 & 0.4414 \\
\hline LBP & Lipopolysaccharide-binding protein & LBP & 0.42 & 0.0004 & 0.78 & 0.7084 \\
\hline LG3BP & Galectin-3-binding protein & LGALS3BP & 0.23 & 0.0006 & 1.29 & 0.8776 \\
\hline PGRP2 & $\mathrm{N}$-acetylmuramoyl-L-alanine amidase & PGLYRP2 & 0.82 & 0.0497 & 1.15 & 0.4157 \\
\hline PLF4 & Platelet factor 4 & PF4 & 1.00 & 0.9667 & 1.41 & 0.0366 \\
\hline PON1 & Serum paraoxonase/arylesterase 1 & PON1 & 0.88 & 0.4606 & 1.42 & 0.0442 \\
\hline
\end{tabular}


Table 3 continued

\begin{tabular}{|c|c|c|c|c|c|c|}
\hline \multirow[t]{2}{*}{ Protein name } & \multirow[t]{2}{*}{ Protein full name } & \multirow[t]{2}{*}{ Gene name } & \multicolumn{2}{|c|}{ Control/cancer } & \multicolumn{2}{|c|}{ Local/metastatic } \\
\hline & & & Ratio & $p$ value & Ratio & $p$ value \\
\hline PROP & Properdin & CFP & 1.45 & 0.0028 & 1.11 & 0.9825 \\
\hline PROZ & Vitamin K-dependent protein Z & $\mathrm{PROZ}$ & 0.93 & 0.6864 & 2.08 & 0.0186 \\
\hline S10A9 & Protein S100-A9 & S100A9 & 0.11 & 0.0054 & 0.74 & 0.2526 \\
\hline SAA1 & Serum amyloid A-1 protein & SAA1 & 0.04 & 0.0407 & 0.49 & 0.2099 \\
\hline SAA2 & Serum amyloid A-2 protein & SAA2 & $<0.01$ & 0.0389 & 0.44 & 0.3069 \\
\hline SEPP1 & Selenoprotein P & SEPP1 & 1.72 & 0.1292 & 1.52 & 0.0186 \\
\hline SHBG & Sex hormone-binding globulin & SHBG & 0.20 & 0.0329 & 0.67 & 0.1968 \\
\hline THBG & Thyroxine-binding globulin & SERPINA7 & 0.70 & 0.0309 & 0.80 & 0.8088 \\
\hline TSP1 & Thrombospondin-1 & THBS1 & 0.74 & 0.1363 & 1.49 & 0.0436 \\
\hline VTDB & Vitamin D-binding protein & GC & 0.81 & 0.0160 & 1.24 & 0.0235 \\
\hline VTNC & Vitronectin & VTN & 0.82 & 0.0394 & 1.15 & 0.1083 \\
\hline
\end{tabular}

Showed are proteins, which abundances were different between samples from healthy controls and patients with stomach cancer (all cases), or between patients with locally advanced and metastatic cancer. Differences (ratios of the mean abundances) that passed the threshold of statistical significance ( $p<0.05$ ) are marked in italics characters

in metastatic samples (Table 3). Furthermore, patterns of differences between samples from patients with metastatic cancer and all patients with local disease were retained when two smaller subgroups of patients with locally advanced cancer were pairwise compared with metastatic cancer (see Additional file 1: Table S4). On the other hand, abundance of only one protein (antithrombin-3) showed statistically significant difference when both subgroups of patients with locally advanced cancer were compared (abundance of this protein was the highest in group of patients with local disease who do not spread during follow-up). We concluded that multiprotein signature could be identified for classification of pre-treatment serum samples of gastric cancer patients, who suffered from either locally advanced or metastatic disease. However, features of serum proteome could not distinguish patients with local disease that spread during follow-up after the treatment.

To identify molecular processes associated with serum proteins characteristic for gastric cancer, corresponding genes were annotated at the Gene Ontology database (Ig-related proteins were excluded from the analysis) (see Additional file 1: Table S5). There were 59 over-represented GO terms associated with proteins differentiating cancer patients from healthy donors. Among them dominated terms associated with different aspects of defense, inflammation and immune response (23 terms). Network of functional interactions was built based on the GeneMANIA Cytoscape tool to illustrate confirmed interactions between serum proteins characteristic for cancer patients. Figure 3 shows such network and its overlap with functional group of proteins associated with inflammatory/acute phase response and/or complement activation (CRP, C1S, CO2, CO4A, CO5, CO6, CO8G, CO9, CFAB, PROP). Moreover, there were 57 over-represented GO terms associated with proteins differentiating patients with locally advanced and metastatic disease. Among them dominated terms associated with metabolism of phosphorus and nucleic acids (16 terms) and with response to cytokines/chemokines and leukocyte migration (13 terms) (see Additional file 1: Table S6). We concluded that serum proteome signature that differentiated gastric cancer patients from healthy donors consisted of proteins primarily involved in cancer-type-nonspecific processes associated with inflammation and immune response (even though Ig-related proteins were excluded from analysis). On the other hand, serum proteome signature characteristic for metastatic cancer consisted of proteins primarily involved in cytokine/chemokine response and/or proliferation.

\section{Discussion}

The last decade has abounded in the publications that reported the MALDI/SELDI-based profiling of the serum peptidome as a promising tool for the effective identification of gastric cancer patients [14-22]. Four different diagnostic classifiers were built on the basis of tri-peptide combination by Ebert et al. [14] (m/z 3946, 3503, 15,958), Su et al. [16] (m/z 1468, 3935, 7560), Liu et al. [19] (m/z 5906.4, 6632.9, 8704.3) and Fan et al. [22] $(\mathrm{m} / \mathrm{z} 1867$, 2701, 2094). Although the discriminatory peaks were not consistent among those studies, probably because of the diverse methodology of sample preparation (especially highly abundant proteins removal), measurement and/or data processing [13], there were some common features of proposed signatures. For example, the $\mathrm{m} / \mathrm{z}$ 


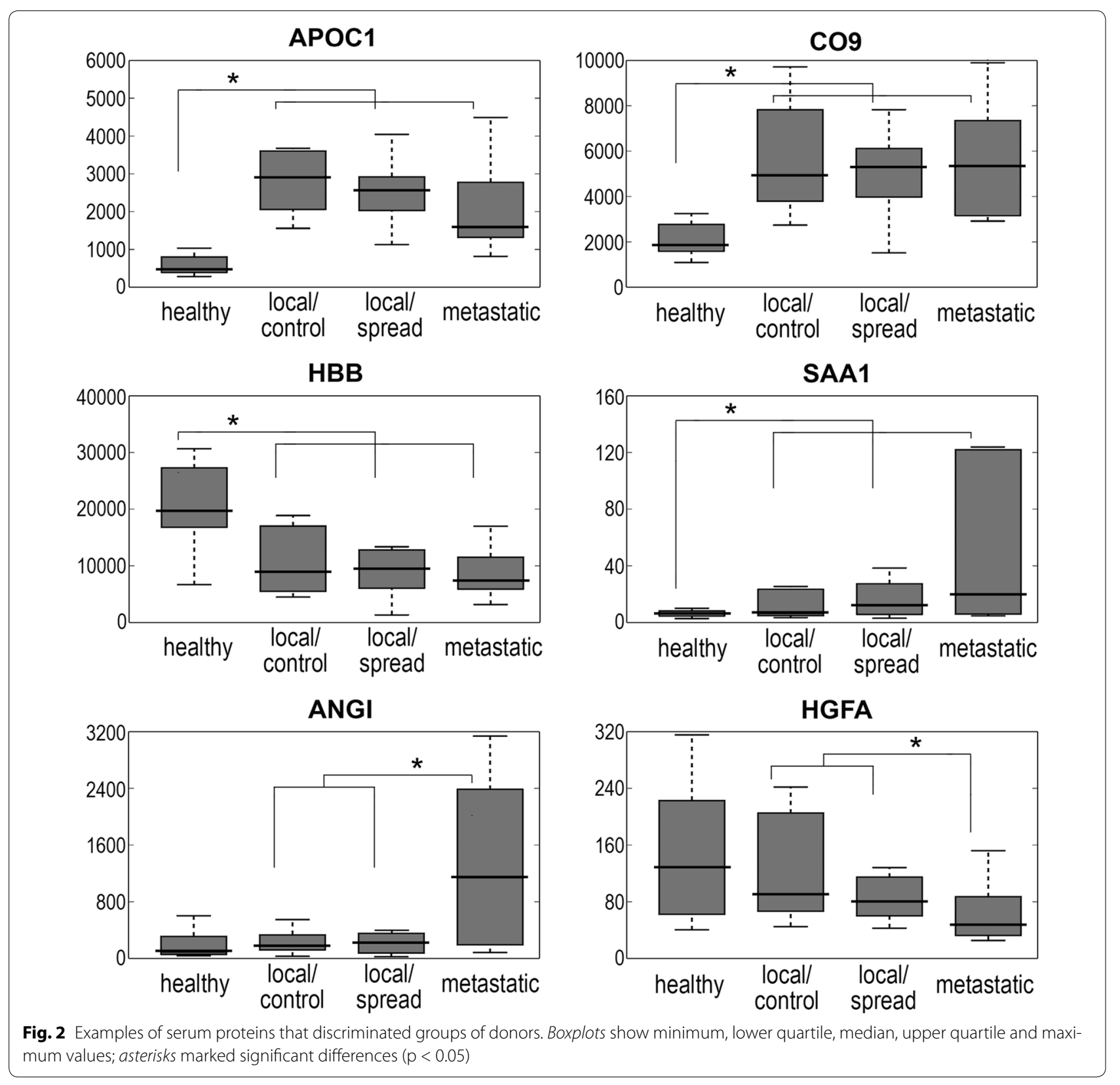

peak at 1466-1468 Da identified as fibrinopeptide A (FIBA) was reported as a candidate biomarker in three reports [16, 23, 24]. Moreover, increased level of another fragment of FIBA (approx. weight 5904-5906 Da) was observed in serum of patients with gastric cancer [19], but also in serum of patients with ovarian, hepatocellular and urothelial cancers [27-29]. In our study we have detected over 100 components of serum peptidome that differentiate compared groups of gastric cancer patients and healthy volunteers. This included several components that putatively corresponded to fragments of FIBA, exemplified by 1469 and 5904 Da components upregulated in blood of patients with metastatic cancer. Thus, our results clearly confirmed and extended previous reports indicating that multipeptide signatures based on features of endogenous serum peptidome could be used for classification of patients with gastric cancer and differentiation of patients with metastatic disease.

In the second part of our study serum samples were further analyzed using the shotgun LC-MS/MS approach, which is currently the gold standard for identification of proteins allowing label-free quantitation and providing 


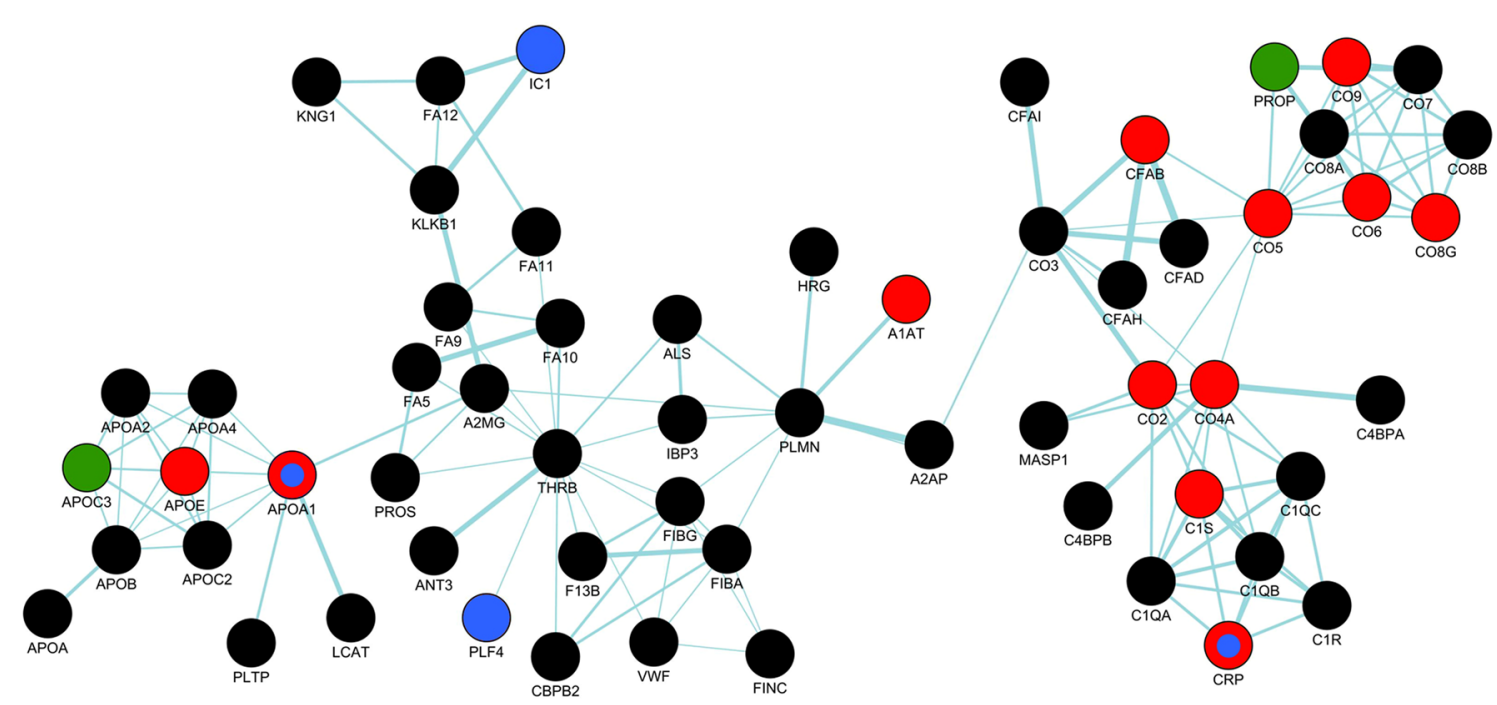

Fig. 3 Network of functional interaction between serum proteins associated with stomach cancer. Red and green circles represent proteins upregulated and downregulated, respectively, in samples from cancer patients; blue circles represent proteins with significantly different abundances in samples of patients with locally advanced and metastatic disease (black circles represent other quantified proteins involved in interactions)

large coverage of sample's proteome [30]. Among serum proteins with abundances significantly different between healthy individuals and patients with gastric cancer dominated those associated with immunity, inflammation and acute phase response, even though immunoglobulins were excluded from the analysis. Up-regulation of proteins involved in immunity and inflammation is a typical picture of serum proteome of cancer patients, especially in advanced cases, and increased levels of proteins like C-reactive protein, haptoglobin or serum amyloid have been previously reported for many different types of cancer [31-35]. Most recently, iTRAQ-based approach has been used to identify serum proteins differentiating healthy controls and patients with gastric adenocarcinoma in a small sample of Asian population [36]. Upregulation of 48 proteins in samples of cancer patients was reported, that included several inflammation/acute phase-related proteins: A1AG1 (ORM1), A1AT (SERPINA1), AACT (SERPINA3), CO4B, CO9, HPT, ITIH4, LBP and SAA1, which upregulation was revealed also in our study. Moreover, other proteins revealed in both reports included upregulated A2GL (LRG1), ITIH3, ORM2 and SHGB, which collectively indicated very high conformity of serum proteome signature of gastric cancer that based on samples of unrelated Polish and India's populations.

Moreover, our study revealed serum proteome components with levels discriminating patients with locally advanced gastric adenocarcinoma and with metastatic disease. Proteins upregulated in serum of patients with metastatic disease included C-reactive protein and IC1
(SERPING1), factors involved in immunity and inflammation, and angiogenin, protein involved in angiogenesis. Other proteins differentially expressed in blood of patients with local and metastatic cancer included molecules associated with response to cytokines/ chemokines, leukocyte migration and blood coagulation as well as factors associated with extracellular transport, and metabolism of phosphorus and nucleic acids. Elevated level of CEA and CA 19-9 was previously associated with increased risk of gastric cancer metastases. However, increased level of such "classical" markers has been observed only in case of $30-50 \%$ of patients with disseminated disease [3-5], thus there is an obvious space for new proteomics-based markers of metastatic gastric cancer. Furthermore, several patients enrolled into the study were diagnosed with locally advanced cancer, yet in some cases the disease was spread and distant metastases were detected during follow-up. However, comparison of pre-treatment serum samples collected in both subgroups of patients with local disease (who further either developed or did not distant metastases) revealed very few significant differences (similar results were delivered by LC-MS/ MS-based analysis of complete proteome and MALDIbased profiling of endogenous peptidome). Hence, our data revealed serum proteome signature discriminating patients with locally advanced and metastatic gastric adenocarcinoma. However, our study did not reveal serum proteome components that could be used for prediction of risk of metastasis in patients diagnosed with local cancer. 


\section{Conclusions}

Significant differences between patients with gastric cancer and healthy individuals as well as between patients with locally advanced and metastatic cancer have been detected at the level of serum proteome in pre-treatment blood samples. However, no evidence that features of pre-treatment serum proteome could predict a risk of cancer dissemination in patients treated due to local disease has been observed. Nevertheless, presented data confirmed potential applicability of biomarkers based on serum proteome signature in diagnostics of gastric cancer.

\section{Additional file}

Additional file 1. Detailed description of differentiating components of serum proteome.

\section{Authors' contributions}

AA designed and performed experiments, drafted manuscript, AW performed MS analyses, AGK performed MS analyses, JP performed mathematical modeling and statistical analyses, PR performed MS analyses, PP collected and interpreted clinical data, ANK collected and interpreted clinical data, MP performed MS analyses, interpreted results, JW designed and interpreted clinical part of study, PW designed overall study, interpreted data and finalized the manuscript. All authors read and approved the final manuscript.

\section{Author details}

${ }^{1}$ Maria Sklodowska-Curie Memorial Cancer Center and Institute of Oncology, Gliwice, Poland. ${ }^{2}$ Silesian University of Technology, Gliwice, Poland. ${ }^{3}$ Institute of Bioorganic Chemistry, Polish Academy of Science, Poznan, Poland.

\section{Acknowledgements}

This work was supported by Polish National Science Centre, Grant No. N403 283140.

\section{Compliance with ethical guidelines}

\section{Competing interests}

The authors declare that they have no competing interests.

Received: 13 June 2015 Accepted: 10 September 2015

Published online: 17 September 2015

\section{References}

1. Ferlay J, Shin HR, Bray F, Forman D, Mathers C, Parkin DM. Estimates of worldwide burden of cancer in 2008: GLOBOCAN 2008. Int J Cancer. 2010;127:2893-917.

2. Hundahl SA, Phillips JL, Menck HR. The National Cancer Data Base report on poor survival of US gastric carcinoma patients treated with gastrectomy: fifth edition American Joint Committee on Cancer staging, proximal disease, and the "different disease" hypothesis. Cancer. 2000;88:921-32.

3. Wobbes T, Thomas CM, Segers MF, Nagengast FM. Evaluation of seven tumor markers (CA 50, CA 19-9, CA 19-9 TruQuant, CA 17-4, CA 195, carcinoembryonic antigen, and tissue polypeptide antigen) in the pretreatment sera of patients with gastric carcinoma. Cancer. 1992;69:2036-41.

4. Kochi M, Fujii M, Kanamori N, Kaiga T, Kawakami T, Aizaki K, et al. Evaluation of serum CEA and CA 19-9 levels as prognostic factors in patients with gastric cancer. Gastric Cancer. 2000;3:177-86.
5. Ucar E, Semerci E, Ustun H, Yetim T, Huzmeli C, Gullu M. Prognostic value of preoperative CEA, CA 19-9, CA 72-4, and AFP levels in gastric cancer. Adv Ther. 2008;25:1075-84.

6. Cancer Genome Atlas Research Network. Comprehensive molecular characterization of gastric adenocarcinoma. Nature. 2014;513:202-9.

7. Leal MF, Assumpcao PP, Smith MC, Burbao RR. Searching for gastric cancer biomarkers through proteomic approaches. J Gastroenterol Hepatol Res. 2014;. doi:10.6051/j.issn.2224-3992.2014.03.363.

8. Liu W, Yang Q, Liu B, Zhu Z. Serum proteomics for gastric cancer. Clin Chim Acta. 2014;431:179-84.

9. Liotta LA, Ferrari M, Petricoin EF. Clinical proteomics: written in blood. Nature. 2003;425:905.

10. Azad NS, Rasool N, Annunziata CM, Minasian L, Whiteley G, Kohn EC. Proteomics in clinical trials and practice: present uses and future promise. Mol Cell Proteom. 2006;5:1819-29.

11. Solassol J, Jacot W, Lhermitte L, Boulle N, Maudelonde T, Mangé A. Clinical proteomics and mass spectrometry profiling for cancer detection. Expert Rev Proteom. 2006;3:311-20.

12. Palmblad M, Tiss A, Cramer R. Mass spectrometry in clinical proteomicsfrom the present to the future. Proteom Clin Appl. 2009;3:6-17.

13. Pietrowska M, Widlak P. MALDI-MS-based profiling of serum proteome: detection of changes related to progression of cancer and response to anticancer treatment. Int J Proteom. 2012;2012:926427.

14. Ebert MP, Meuer J, Wiemer JC, Schulz HU, Reymond MA, Traugott U, et al. Identification of gastric cancer patients by serum protein profiling. J Proteome Res. 2004;3:1261-6.

15. Poon TC, Sung JJ, Schow SM, Ng EK, Yu AC, Chu ES, et al. Diagnosis of gastric cancer by serum proteomic fingerprinting. Gastroenterology. 2006:130:1858-64.

16. Su Y, Shen J, Qian H, Ma H, Ji J, Ma L, et al. Diagnosis of gastric cancer using decision tree classification of mass spectral data. Cancer Sci. 2007; 98:37-43.

17. Qiu FM, Yu JK, Chen YD, Jin QF, Sui MH, Huang J. Mining novel biomarkers for prognosis of gastric cancer with serum proteomics. J Exp Clin Cancer Res. 2009:28:126.

18. Umemura H, Togawa A, Sogawa K, Satoh M, Nishimura M, Matsushita K, et al. Identification of a high molecular weight kininogen fragment as a marker for early gastric cancer by serum proteome analysis. J Gastroenterol. 2011;46:577-85.

19. Liu C, Pan C, Liang Y. Screening and identification of serum proteomic biomarkers for gastric adenocarcinoma. Exp Ther Med. 2012;3:1005-9.

20. Liu C, Pan C, Shen J, Wang H, Young L. Identification of serum amyloid A in the serum of gastric cancer patients by protein expression profiling. Oncol Lett. 2012;3:1259-62.

21. Yang J, Song YC, Dang CX, Song TS, Liu ZG, Guo YM, et al. Serum peptidome profiling in patients with gastric cancer. Clin Exp Med. 2012;12:79-87.

22. Fan NJ, Li K, Liu QY, Wang XL, Hu L, Li JT, Gao CF. Identification of tubulin beta chain, thymosin beta-4-like protein 3, and cytochrome b-c1 complex subunit 1 as serological diagnostic biomarkers of gastric cancer. Clin Biochem. 2013:46:1578-84.

23. Ebert MP, Niemeyer D, Deininger SO, WexT, Knipping C, Hoffmann J, et al. Identification and confirmation of increased fibrinopeptide A serum protein levels in gastric cancer sera by magnet bead assisted MALDI-TOF mass spectrometry. J Proteome Res. 2006;5:2152-8.

24. Zhang MH, Xu XH, Wang Y, Ling QX, Bi YT, Miao XJ, et al. A prognostic biomarker for gastric cancer with lymph node metastases. Anat Rec (Hoboken). 2013;296:590-4.

25. Humphries JM, Penno MA, Weiland F, Klinger-Hoffmann M, Zuber A, Boussioutas A, et al. Identification and validation of novel candidate protein biomarkers for the detection of human gastric cancer. Biochim Biophys Acta. 2014;1844:1051-8.

26. Lustgarten JL, Kimmel C, Ryberg H, Hogan W. EPO-KB: a searchable knowledge base of biomarker to protein links. Bioinformatics. 2008;24:1418-9.

27. Bergen HR 3rd, Vasmatzis G, Cliby WA, Johnson KL, Oberg AL, Muddiman DC. Discovery of ovarian cancer biomarkers in serum using NanoLC electrospray ionization TOF and FT-ICR mass spectrometry. Dis Markers. 2003;19:239-49. 
28. Orvisky E, Drake SK, Martin BM, Abdel-Hamid M, Ressom HW, Varghese $\mathrm{RS}$, et al. Enrichment of low molecular weight fraction of serum for MS analysis of peptides associated with hepatocellular carcinoma. Proteomics. 2006:6:2895-902.

29. Theodorescu D, Wittke S, Ross MM, Walden M, Conaway M, Just I, et al. Discovery and validation of new protein biomarkers for urothelial cancer: a prospective analysis. Lancet Oncol. 2006;7:230-40.

30. Vaudel M, Sickmann A, Martens L. Current methods for global proteome identification. Expert Rev Proteom. 2012;9:519-32.

31. Firpo MA, Gay DZ, Granger SR, Scaife CL, DiSario JA, Boucher KM, et al. Improved diagnosis of pancreatic adenocarcinoma using haptoglobin and serum amyloid A in a panel screen. World J Surg. 2009;33:716-22.

32. Cho WC, Yip TT, Cheng WW, Au JS. Serum amyloid A is elevated in the serum of lung cancer patients with poor prognosis. Br J Cancer. 2010;102:1731-5.
33. Cocco E, Bellone S, El-Sahwi K, Cargnelutti M, Buza N, Tavassoli FA, et a Serum amyloid A: a novel biomarker for endometrial cancer. Cancer. 2010;116:843-51.

34. Hong T, Liu A, Cai D, Zhang Y, Hua D, Hang X, et al. Preoperative serum C-reactive protein levels and early breast cancer by BMI and menopausal status. Cancer Invest. 2013;31:279-85.

35. Trabert B, Pinto L, Hartge P, Kemp T, Black A, Sherman ME, et al. Pre-diagnostic serum levels of inflammation markers and risk of ovarian cancer in the prostate, lung, colorectal and ovarian cancer (PLCO) screening trial. Gynecol Oncol. 2014;135:297-304

36. Subbannayya Y, Mir SA, Renuse S, Manda SS, Pinto SM, Puttamallesh VN, et al. Identification of differentially expressed serum proteins in gastric adenocarcinoma. J Proteom. 2015;. doi:10.1016/j.jprot.2015.04.021.
Submit your next manuscript to BioMed Central and take full advantage of:

- Convenient online submission

- Thorough peer review

- No space constraints or color figure charges

- Immediate publication on acceptance

- Inclusion in PubMed, CAS, Scopus and Google Scholar

- Research which is freely available for redistribution

Submit your manuscript at

www.biomedcentral.com/submit 